\title{
Formability Effects of Variable Blank Holder Force on Deep Drawing of Stainless Steel
}

\author{
Pramote Koowattanasuchat ${ }^{1}$, Numpon Mahayotsanun ${ }^{1}$, Sukunthakan Ngernbamrung ${ }^{2}$, and Sasawat Mahabunphachai ${ }^{2, a}$ \\ ${ }^{1}$ Department of Mechanical Engineering, Faculty of Engineering, Khon Kaen University, 123 Moo 16 Mittraphap Road, Nai-Muang, Muang \\ District, Khon Kaen 40002 Thailand \\ ${ }^{2}$ National Metal and Materials Technology Center (MTEC), 114 Thailand Science Park, Phahonyothin Road, Klong 1, Klong Luang, \\ Phathumthani 12120 Thailand
}

\begin{abstract}
This paper investigates the formability effects of variable blank holder force on deep drawing of AISI 304 rectangular cup. Various sets of blank holder forces were set to observe the formability, which was indicated by the percentage of sheet thinning in this study. The results showed that the blank holder forces at the locations of the sheet edges surrounding the cavity areas were considered dominant to reduce sheet thinning and enhance the formability.
\end{abstract}

\section{Introduction}

A recent trend in deep drawing of stainless steel sheets is to acquire deep drawn depths, sharp corners, and highly sophisticated shapes in order to add value to the products. To successfully form such parts, understanding and controlling the flow of the sheet material are critical.

Many techniques have been utilized in deep drawing to enhance material flow, for instances, forming at elevated temperatures, annealing between stamping steps, electric shimming, applying ultrasonic vibration, etc. Using variable blank holder force (BHF) is also a common technique to effectively control the material flow and enhance the formability [1-10]. This paper investigates the formability effects under different blank holder forces on deep drawing of AISI 304 rectangular cup. Various sets of blank holder forces were applied to observe the effect of sheet formability, which was indicated by the sheet thinning percentage. The results and analysis of this study could be used to acquire optimized sets of blank holder forces that would yield good formability.

\section{Deep drawing experiment}

The experimental setup of the deep drawing experiment using variable blank holder force control carried out in this study is illustrated in Figure 1. There were three sets (A, B, and C) of variable blank holder force located at set positions as shown in that figure. These variable blank holder forces were pressure-controlled by nitrogen-gas springs. In this study, nine variable blank holder force conditions were considered as shown in Figure 2. The values of the set pressure were ranged from low (L, 50 Bar) to medium (M, $100 \mathrm{Bar}$ ) to high (H, $150 \mathrm{Bar}$ ).

In this study, the sheet dimensions were $337.5 \mathrm{~mm}$ x $237.0 \mathrm{~mm} \times 0.4 \mathrm{~mm}$ with the longitudinal direction parallel to the rolling direction. Since AISI 304 sheet under the annealed condition is quite isotropic [11], the effect of anisotropy on the formability of the sheet is neglected in this study. The punch dimensions were 99.6 $\mathrm{mm} \times 112.1 \mathrm{~mm} \times 90.9 \mathrm{~mm}$. The die dimensions were $347.5 \mathrm{~mm} \times 440.0 \mathrm{~mm} \times 20 \mathrm{~mm}$. The blank holder dimensions were $347.5 \mathrm{~mm}$ x $440.0 \mathrm{~mm}$ x $30 \mathrm{~mm}$. The tool (punch, die and blank holder) material was SKD11 and the sheet material was AISI 304. The mechanical properties of the considered tool and sheet materials are presented in Table 1. Prior to each deep drawing test, a lubricant was applied on both sides of a sheet. The properties of the lubricant used in this study are presented in Table 2. During the test, a lubricated sheet was placed in between the blank holder and the die. The die was then moved vertically downward for $30 \mathrm{~mm}$ to form the sheet into the rectangular cup shape.

In order to investigate the formability under different variable blank holder forces, the percentage of thinning of sheet thickness after being deep drawn was used as an indicator. After each test, the sheet thickness at three measuring lines $\left(0^{\circ}, 45^{\circ}\right.$ and $\left.90^{\circ}\right)$ was measured at 10 locations as illustrated in Figure 3. The percentage of thinning can be calculated by Equation 1 .

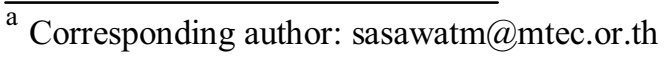




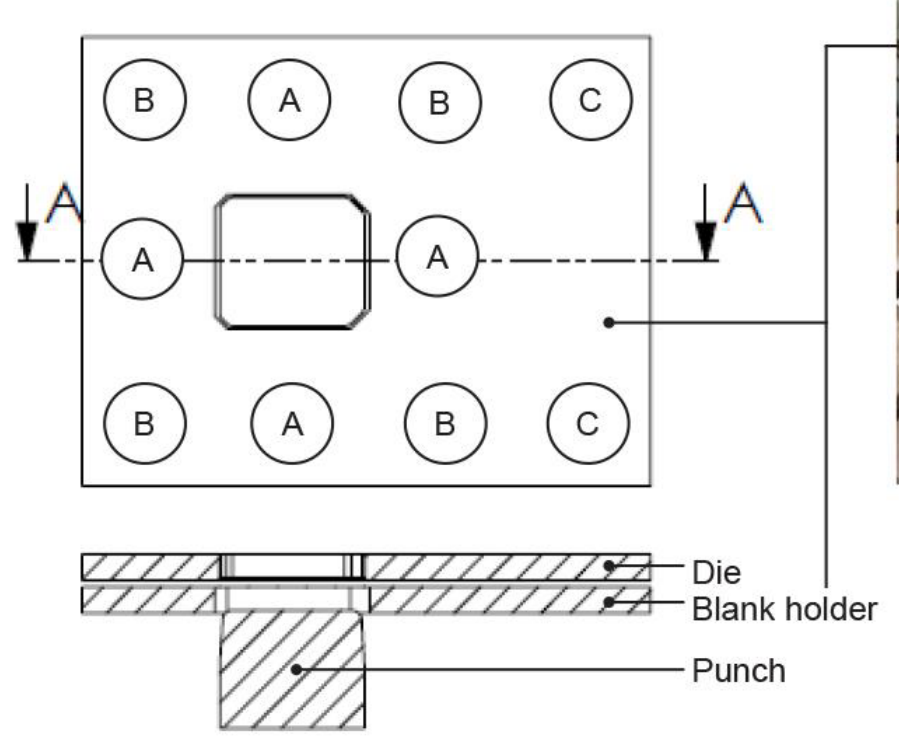

SECTION A-A

Figure 1. Deep drawing setup using variable blank holder force control.
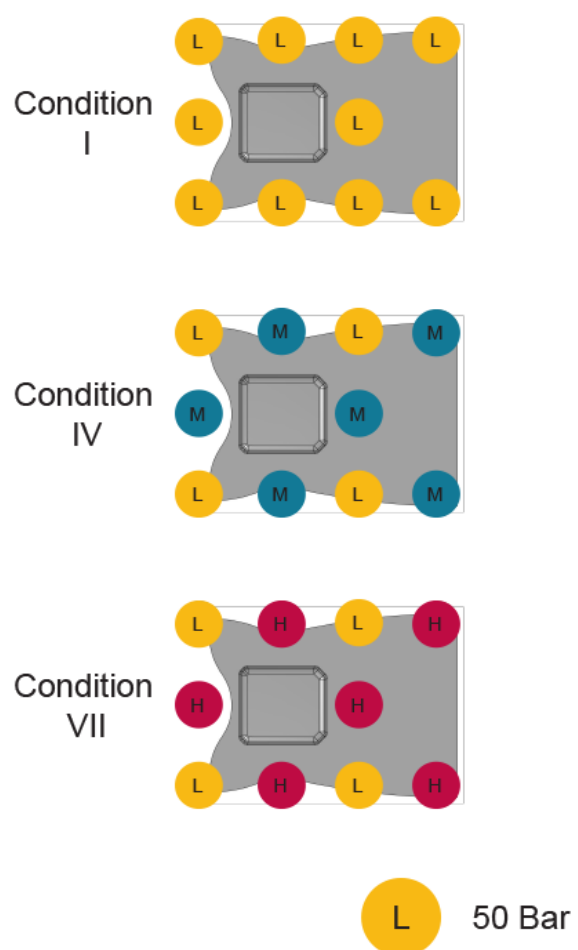
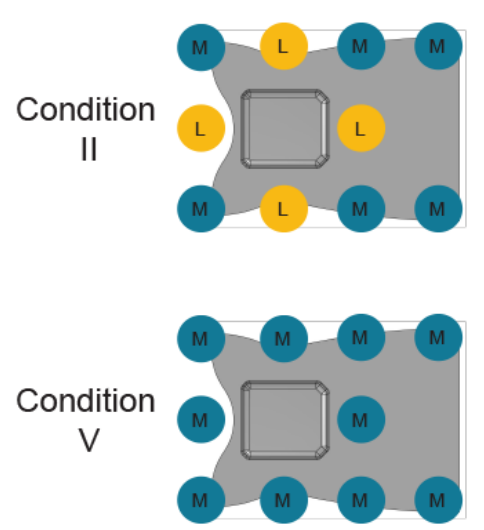
(A) Gas springs group A
(B) Gas springs group B
(C) Gas springs group C
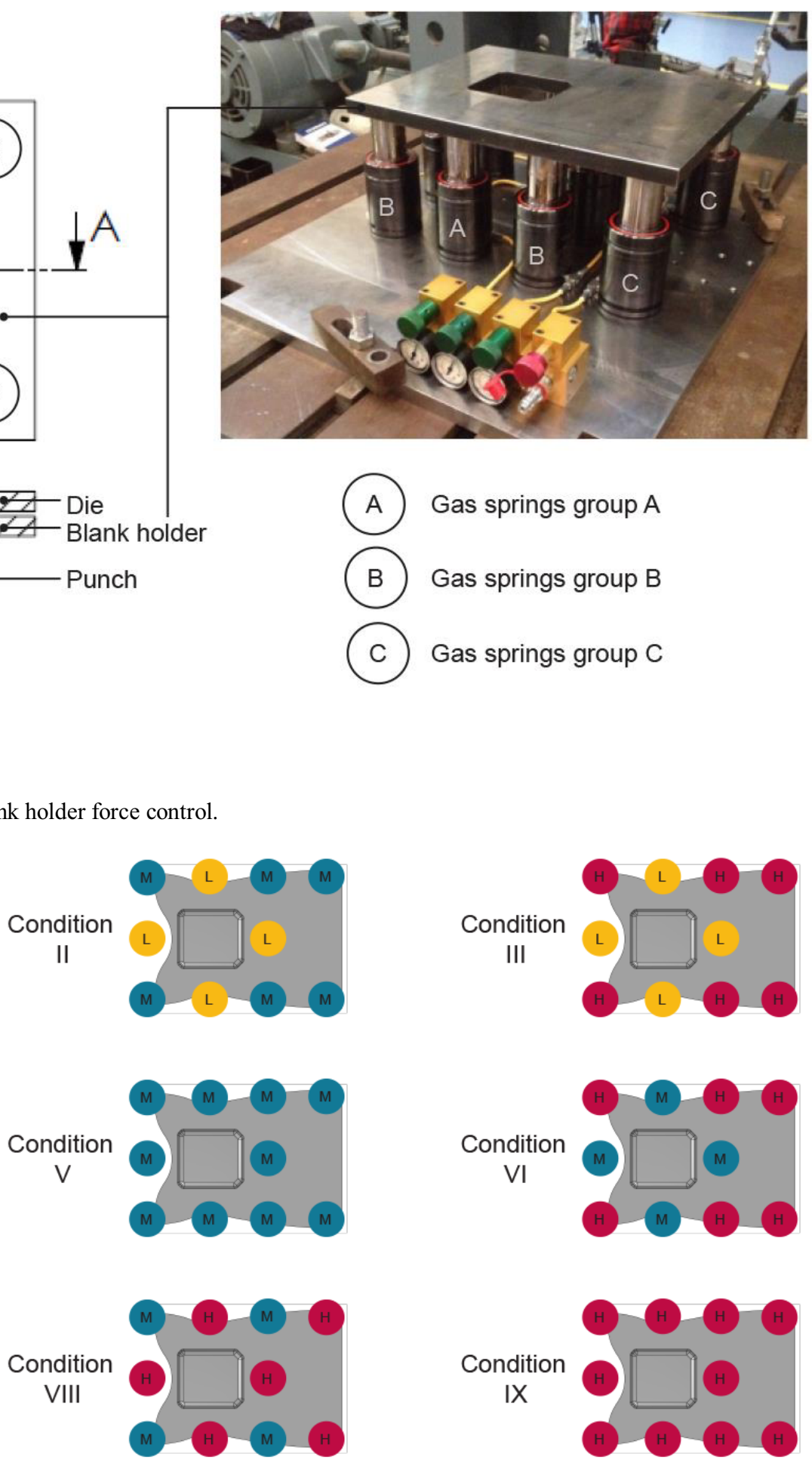

Figure 2. Considered variable blank holder force conditions.

Table 1. Mechanical properties of the considered tool and sheet materials.

\begin{tabular}{|c|c|c|c|c|c|}
\hline Part & Material & $\begin{array}{c}\text { Tensile } \\
\text { strength } \\
\left(\mathbf{N} / \mathbf{m m}^{2}\right)\end{array}$ & $\begin{array}{c}\text { Yield } \\
\text { strength } \\
\left(\mathbf{N} / \mathbf{m m}^{2}\right)\end{array}$ & $\begin{array}{c}\text { Elongation } \\
\mathbf{( \% )}\end{array}$ & $\begin{array}{c}\text { Hardness } \\
\text { (HRC) }\end{array}$ \\
\hline Tool & SKD11 & - & - & - & $58-62$ \\
\hline Sheet & AISI 304 & 734 & 305 & 50 & - \\
\hline
\end{tabular}


Table 2. Properties of the lubricant used in this study.

\begin{tabular}{|c|c|c|c|c|c|c|}
\hline Active elements & $\begin{array}{c}\text { Base oil } \\
\text { group }\end{array}$ & Appearance & $\begin{array}{c}\text { Specific } \\
\text { gravity } \\
\left(\mathbf{g} / \mathbf{c m}^{\mathbf{3}} \mathbf{1 5}^{\mathbf{0}} \mathbf{C}\right)\end{array}$ & $\begin{array}{c}\text { Viscosity } \\
\left(\mathbf{m m}^{\mathbf{2}} / \mathbf{s} \mathbf{4} \mathbf{4 0}^{\mathbf{}} \mathbf{C}\right)\end{array}$ & $\begin{array}{c}\text { Copper } \\
\text { corrosion test } \\
\left(\mathbf{1 0 0}^{\mathbf{0}} \mathbf{C} \times \mathbf{1 h r}^{\mathbf{h}}\right)\end{array}$ & Application \\
\hline $\begin{array}{c}\text { Chlorine }(<20 \mathrm{wt} \%), \text { Sulfur } \\
(<10 \mathrm{wt} \%), \text { Fatty oil }(<20 \\
\mathrm{wt} \%)\end{array}$ & $\begin{array}{c}\text { Group } \\
\text { II }\end{array}$ & $\begin{array}{c}\text { Dark red } \\
\text { transparent }\end{array}$ & 1.0 & 135 & 2 (Inactive) & Forming oil \\
\hline
\end{tabular}

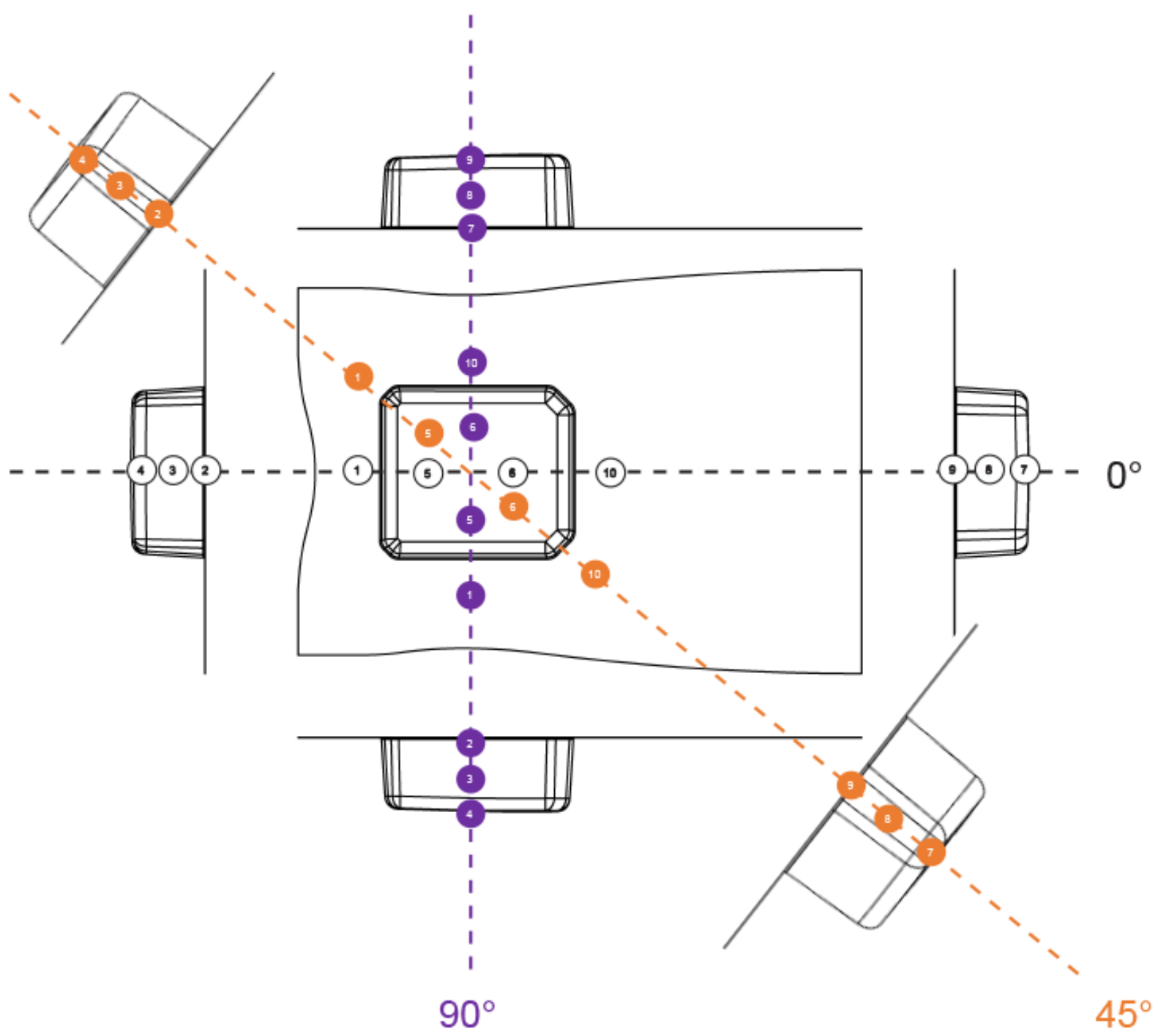

Figure 3. Sheet thickness measurement locations after deep drawing tests.

$$
T_{\%}=\left(T_{\text {initial }}-T_{\text {final }}\right) \times 100 \% / T_{\text {initial }}
$$

where $\left(T_{\%}\right)$ is the percentage of thinning, $\left(T_{\text {initial }}\right)$ is the initial sheet thickness, and $\left(T_{\text {final }}\right)$ is the final sheet thickness.

\section{Results and discussions}

The results of percentage of thinning of all the conditions are shown in Figure 4. In each graph, the calculated percentage of thinning values was plotted against measured points (10 locations) at three measured lines $\left(0^{\circ}, 45^{\circ}, 90^{\circ}\right)$. Although none of the conditions provided any defects (tearing or wrinkling) after the deep drawing tests, the locations where the values of percentage of thinning were high (reduced sheet thickness) might lead to tearing if higher drawn depths were required. According to the results, it could be observed that the locations number 8 were prone to thinning because the nearby areas (locations number 7 and locations number 9) were the cup corners, restricting the material flow and causing the sheet to elongate with reduced thickness. Location numbers 1 and 10 provided the lowest percentage of thinning values due to the fact that these areas were tightly compressed between the die and blank holder, prohibiting the sheet to move and deform. 
In order to observe the effect of blank holder force, particularly at the locations prone to thinning (numbers 7 , 8 and 9), nine various sets of conditions were compared as follows:

- Conditions I, II and III. The main difference among these three conditions is the increased blank holder forces in Groups B and C from Condition I, to Condition III, respectively while blank holder forces in Group A were kept at 50 Bar (Low). It could be observed that the percentage of thinning at locations number 7,8 and 9 tended to reduce if the variable blank holder forces increased from 50 Bar (Condition I) to 100 Bar (Condition II), indicating that increasing the blank holder force in the sheet edges allowed the sheet to flow well if the blank holder forces at the cavity edges were kept low. However, increasing the variable blank holder forces from $100 \mathrm{Bar}$ (Condition II) to 150 Bar (Condition III) did not further decrease the percentage of thinning.

- Conditions I, IV and VII. The main difference among these three conditions is the increased blank holder forces in Groups A and C from Conditions I, IV and VII, respectively while blank holder forces in Group B were kept at 50 Bar (low). It could be observed the no significant effect could be observed through the variations of blank holder forces in the areas close to the cavity edges.

- Conditions IV, $V$ and $V I$. The main difference among these three conditions is the increased blank holder forces in Groups B and C from Conditions I, to Condition III, respectively while blank holder forces in Group A were kept at medium (100 Bar). It could be observed that the percentage of thinning at locations number 7,8 and 9 tended to reduce if the variable blank holder forces increased from 50 Bar (Condition IV) to 100 Bar (Condition V), indicating that increasing variable blank holder force in the sheet edges allowed the sheet to flow well if the blank holder forces at the cavity edges were kept medium. However, increasing the variable blank holder forces from 100 Bar (Condition V) to 150 Bar (Condition VI) did not further decrease the percentage of thinning.

- Conditions VII, VIII and IX. The main difference among these three conditions is the increased blank holder forces in Groups B and C from Conditions VII, to Condition IX, respectively while blank holder forces in Group A were kept a 150 Bar (High). It could be observed that the percentage of thinning at locations number 7,8 and 9 tended to reduce if the variable blank holder forces increased from 50 Bar (Condition VII) to 100 Bar (Condition VIII), indicating that increasing variable blank holder force near the sheet edges allowed the sheet to flow easier into the die cavity when the blank holder forces at the cavity edges were kept medium. However, increasing the blank holder forces from 100 Bar
(Condition VIII) to 150 Bar (Condition IX) was increase the percentage of thinning.

- Conditions III, VI and IX. The main differences among these three conditions were the increased blank holder forces in Groups A from Condition III, VI and IX, respectively while blank holder forces in Groups B and C were kept at 150 Bar (High). No significant effect is observed through the variations of blank holder forces in the areas close to the cavity edges if the blank holder forces at the sheet edges were kept high.

- Conditions I, $V$ and IX. The main difference among these three conditions is the overall increased blank holder forces in Groups A, B and C from Conditions I, V and IX, respectively. No significant effect is observed through the increased of the overall blank holder forces in all the areas.

According to the comparisons of the aforementioned conditions, it is observed that increasing the blank holder force at the sheet edges from low (50 Bar) to medium (100 Bar) tended to reduce the percentage of thinning. However, increasing the blank holder force further from medium (100 Bar) to high (150 Bar) did not decrease the percentage of thinning further. In addition, increasing the blank holder force at the cavity edges did not significantly affect the percentage of thinning.

In order to observe the effect of variable blank holder force to all the locations (numbers 1 to 10 ), the percentage of thinning of locations number 1 to 10 were averaged and plotted against all the conditions as shown in Figure 5. The graphs of the left to right columns show the ranges of the percentage of thinning at $0^{\circ}, 45^{\circ}, 90^{\circ}$ and the average (mean of $0^{\circ}$, $45^{\circ}$, and $90^{\circ}$ ), respectively. It could be clearly seen that the average values of percentage of thinning measured at $90^{\circ}$ is the lowest compared to those of $0^{\circ}$ and $45^{\circ}$. This could be due to the fact that the rectangular cup examined in this study provided more areas for the sheet material to flow in the $90^{\circ}$ direction than those of $0^{\circ}$ and $45^{\circ}$ directions. By observing the values of the percentage of thinning of average (mean of $0^{\circ}, 45^{\circ}$, and $90^{\circ}$ ), it could be noticed that Conditions II, V and VIII provided low percentage of thinning values. These are the conditions where the blank holder forces of Group B are kept at medium value (100 Bar). Group B controlled the blank holder locations of the sheet edges surrounding the cavity areas, which was considered the dominant set of locations to control the variable blank holder forces.

In order to obtain the optimum amounts and locations of blank holder forces, a finite element simulation and theoretical modelling are currently being carried out by the authors. However, the analysis of the simulation results and theoretical models will be published in a future work. Nevertheless, the experimental results obtained from this study can still be used as a guideline to design and select the appropriate distribution blank holder forces that can reduce sheet thinning and enhance the formability. 

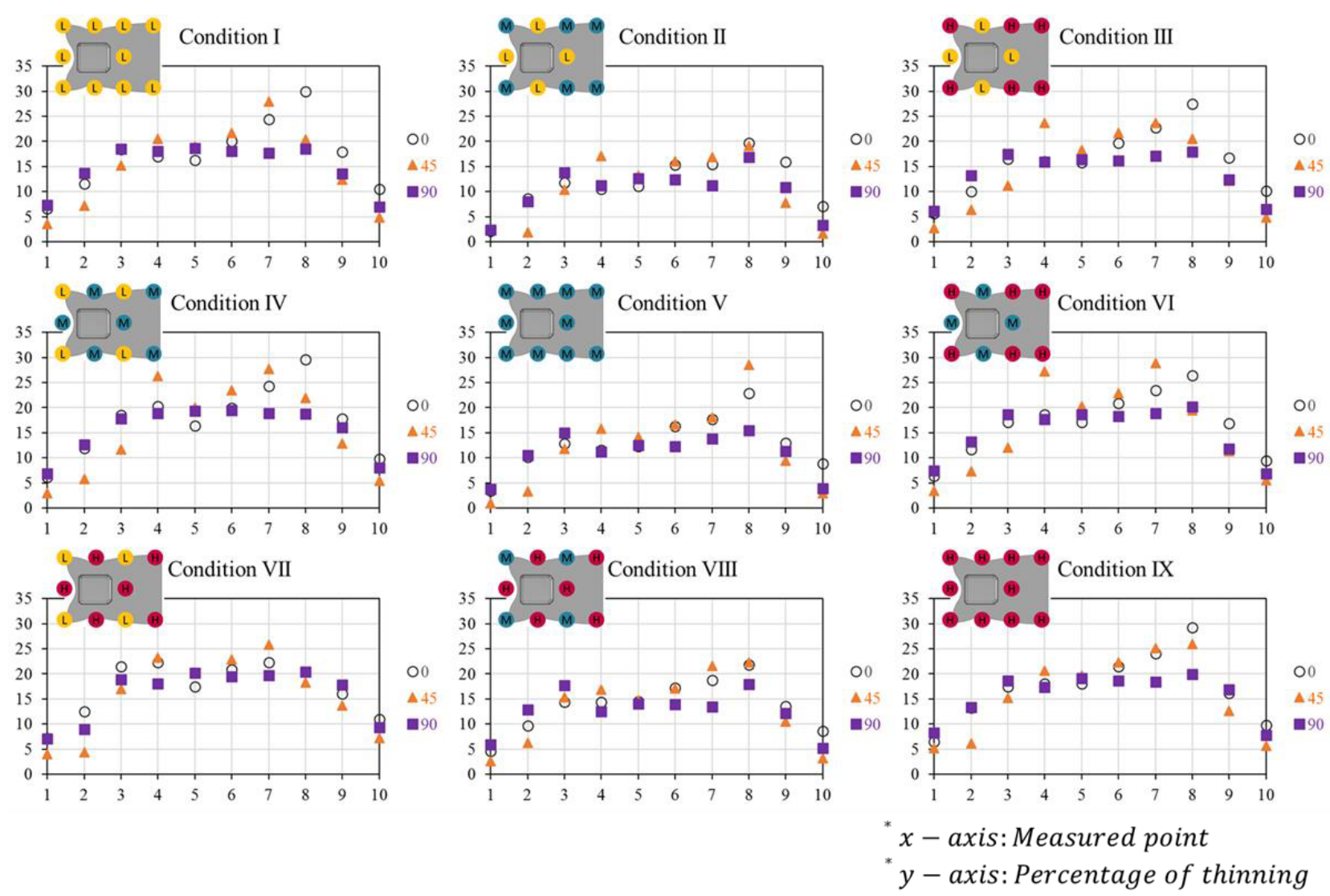

Figure 4. Percentage of thinning vs. measured points of all the considered conditions.
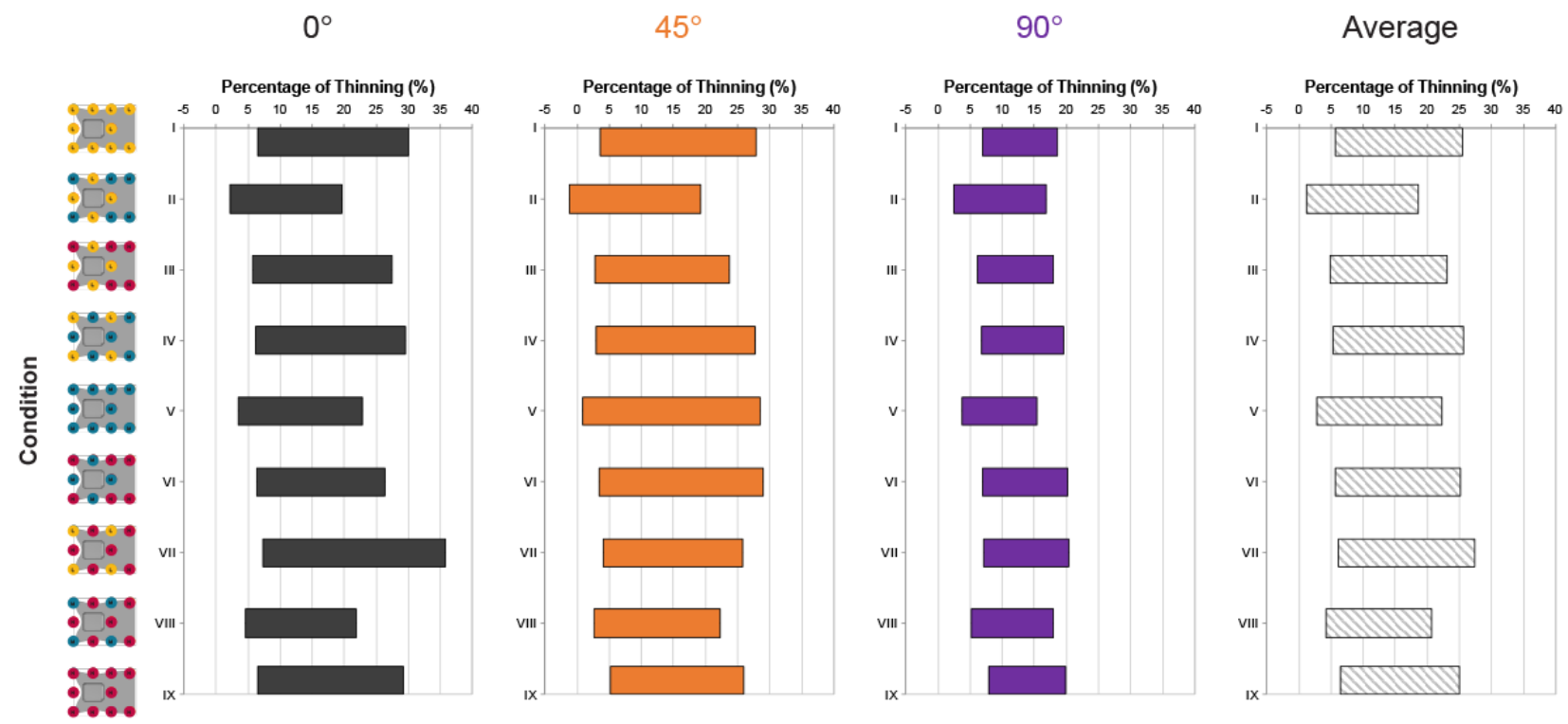

Figure 5. Average percentage of thinning of all the considered conditions.

\section{Conclusions}

This study experimentally investigated the effect of the distribution of the blank holder forces on the formability of AISI 304 in the deep drawing under lubricated conditions. The ranges of blank holder forces ranged from 50 Bar to 150 Bar and nine conditions of blank holder forces were configured under three groups of pressure-controlled positions. The sheet thickness values after the deep drawing tests were measured at three set lines $\left(0^{\circ}, 45^{\circ}\right.$ and $\left.90^{\circ}\right)$ each having 10 different points. The formability of each condition was observed through the percentage of sheet thinning. The results showed that the blank holder forces at the locations of the sheet edges surrounding the box corner areas are found dominant to reduce the percentage of thinning and thus enhance the formability.

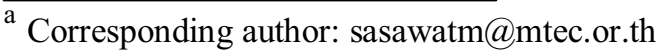




\section{Acknowledgement}

The study was financially supported by the NSTDA University Industry Research Collaboration (NUI-RC). The authors would also like to acknowledge the supports of Diamond Brand Co., Ltd., National Metal and Materials Technology Center (MTEC), and Department of Mechanical Engineering, Faculty of Engineering, Khon Kaen University.

\section{References}

1. W.-r. Wang, G.-1. Chen and Z.-q. Lin, Trans. Nonferrous Met. Soc. China 20, 471 (2010).

2. S. Qin, L. Yang and B. Yang, ICA. 2, 450-455 (2011).

3. H. Koyama, R. H. Wagoner and K.i. Manabe, J. Mater. Process. Technol. 152, 190 (2004).

4. S.-x. Tian, Y.-x. Feng and Y.-c. Gao, Procedia CIRP 27, 309 (2015).

5. L. Zhong-qin, W. Wu-rong and C. Guan-long, J. Mater. Process. Technol. 183, 339 (2007).

6. B. Endelt, S. Tommerup and J. Danckert, J. Mater. Process. Technol. 213, 36 (2013).

7. L. B. Shulkin, R. A. Posteraro and M. A. Ahmetoglu, J. Mater. Process. Technol. 98, 7 (2000).

8. T. Intarakumthornchai, S. Jirathearanat, S. Thongprasert and P. Dechaumphai, APIEMS Conference (2006).

9. H. Koyama, R. H. Wagoner and K.i. Manabe, J. Mater. Process. Technol. 152, 190 (2004).

10. E.J. Obermeyer and S. A. Majlessi, J. Mater. Process. Technol. 75, 222 (1998).

11. H. G. Steyn, J. Mater. Eng. Perform. 21, 1491 (2012) 